\title{
ACTIVIDADES DEL DEPARTAMENTO
}

\section{CREACION DE LA CARRERA DE LICENCIATURA EN MATEMATICA}

Por decreto $343 / 81$ de Julio del año 1981 se oficializaron las carreras nuevas que ofrece la Universidad del Norte a partir del presente año. En dicho decreto, se crea entre otras la carrera de "Licenciatura en Matemáticas", otorgando el grado de Licenciado y los títulos de Matemático o Profesor de Matemática.

E1 Licenciado en Matemática es un profesional, con sólidos conocimientos en las principales ramas de las Matemáticas, con la suficiente capacidad en el uso y aplicación de los métodos propios de estas ramas, 1o que 1o habilita para desempeñarse en la docencia superior de pregrado o como colaborador en grupos de investigadores, tanto de Matemática Pura como Aplicada. En este último caso 
puede participar en problemas de la planificación o diseño industrial.

E1 martes 27 de Abril del presente, se realizó la clase inaugural de la carrera de Licenciatura en Matemáticas dictada por el Profesor Titular y Director del Instituto de Matemática de la Pontificia Universidad Católica de Chile, Dr. Rolando Chuaqui K.. En dicha clase el Dr. Chuaqui expuso la importancia de la investigación matemática y la urgente necesidad de la formación de Matemáticos.

La creación del Programa de Licenciatura en Matemática en nuestra Universidad, abre una nueva etapa en el desarrollo matemático de nuestra Región. Un programa con exigencia matemática significa poner en práctica, en su totalidad y a fondo el perfeccionamiento logrado por los profesores que sirven actualmente en el Departamento, como también mantener y aumentar este perfeccionamiento; preparación adecuada para futuros académicos; contacto e integración con los diferentes centros de Enseñanza Superior del país y del extranjero y posibilidades de investigación en diferentes campos de la Matemática. La Licenciatura es el primer paso hacia programas más elevados como el de Magister y Doctorado.

En este primer semestre la Licenciatura en Matemática tiene 50 alumnos $y$ se dictan, para ellos las asignaturas de Geometría I; Algebra I, Geometría Analítica, y además, dos ramos de Formación General. 


\section{INVESTIGACION UNIVERSITARIA.}

A partir de Marzo de 1981, se están desarrollando dos proyectos de investigación en el Departamento de Matemática de la Universidad del Norte.

1. Tratamiento bayesiano para algunos juegos, a cargo de los docentes: Arnoldo Prado Campos (Doctor en Estadística), Raúl Zhigley Correa (Master en Estadística) y Patricio Rojas Morera (Máster en Estadística).

Estas investigación se base en el hecho conocido de que en sectores de la economía y otros de las ciencias aplicadas hay situaciones en las que los modelos probabilísticos adquieren una mayor amplitud y profundidad cuando se abordan desde un punto de vista bayesiano. Este es el caso de los juegos de estrategias cuando existe incertidumbre respecto a las funciones de pago o en términos más generales con respecto a los parámetros que definen el juego. Específicamente la investigación abordará el caso de un juego m-personal, m-cooperativo cuando cada jugador dispone de un equipo de informadores de las decisiones que adopte su contrincante y la información tiene sólo una probabilidad positivo menor que uno, de ser veraz.

2. Estudio del Algoritmo de Euclides (Minimal) en ciertos anillos, a cargo de los docentes: Rodolfo Baeza Vega (Magíster en Matemática, candidato a Doctor), y María Soledad Romo López (Magister en Matemática, candidata a Doctor).

Se pretende encontrar al algoritmo minimal para algunos anillos especiales, por ejemplo $\mathrm{Z}(\sqrt{-2})$ y acotar 
más finamente el número de etapas necesarios para calcular el máximo común divisor en un Dominio Euclídeo.

Desde Mayo de 1982, ha comenzado a desarrollarse el proyecto Solución numérica de la ecuación de Poisson, a cargo de los docentes: Oscar Rojo Jeraldo (Doctor en Matemáticas), Ricardo Soto Montero (Magister en Matemática), Luis Valdivia Reyes (Magister en Matemática).

La investigación propuesta pretende:

1. Estudiar y comparar diferentes métodos, para resolver numéricamente la ecuación de Poisson en un rectángulo con condiciones de frontera de Dirichlet, Newman y periódicas, y su extensión a regiones no rectangulares. En particular, interesa el estudio de los métodos Hockney y Buneman, Fourier-Toeplitz, Métodos iterativos. En estos últimos aparece el método de sobre-relajación acelerada, de reciente publicación en la literatura matemática. Interesa, en especial, aplicar dicho método a la Ecuación de Poisson y estudiar sus posibles ventajas.

2. Implementar programas computacionales para los diferentes métodos.

3. Eventualmente encontrar modificaciones a algún método que lo haga más eficiente. 


\section{PROGRAMAS DE EXTENSION}

E1 Departamento de Matemática de nuestra Universidad, se encuentra en estos momentos abocado al desarro110 de 4 programas de extensión que son: Academia de Matemáticas destinada a alumnos de Enseñanza Media; Edición de una Revista de Matemática a nivel informativo, organización de la Primera Jornada Matemática de la Universidad del Norte y una investigación sobre los Requisitos de Ingreso a la Universidad.

Con respecto a la formación de Academia de Matemática para alumnos de Enseñanza Media, ella está dedicada para quienes en este nivel de enseñanza demuestren inclinaciones matemáticas. Tratará temas no consignados en sus planes de estudio, y que pudieran contribuir a incentivar sus inclinaciones y difundir el conocimiento matemático tales como: Lógica Matemática, Estadística descriptiva, Programación en Computación, Historia de las Matemáticas,Aplicación de la Estadística, Algebra Booleana, etc..

Este programa se realiza en dos etapas, la lera. de Abril a Julio de 1982, y la 2da. de Agosto a Diciembre de 1982 .

En cuanto a la Revista de Matemática, ella será de frecuencia trimestral, estará destinada a todo lector, y contendrá temas de interés científico e informativo de la actividad que se desarrolla en estos momentos en nuestro Departamento.

Consideramos que la labor que se está realizando 
Necesita ser difundida. En ella nuestros académicos podrán adquirir experiencia en la confección de artículos de corte científico, destinados a todo lector.

Estas publicaciones se realizarán en Junio, Septiembre y Diciembre de 1982.

En relación a la "Primera Jornada de Matemáticas de la Universidad del Norte", ella se llevará a efecto en forma de cursillos y charlas dictadas por los profesores invitados y profesores de nuestro Departamento. Esta jornada se realizará entre el 2 y 7 de Agosto, finalizando con un Encuentro Matemático auspiciado y organizado por 1a "Sociedad de Matemática de Chile".

Los principales objetivos de esta jornada son los de divulgar trabajos originales en que se efectúan en nuestro Departamento, por medio de charlas y exposiciones; y el de actualizar la formación matemática de profesionales de la zona norte, mediante la entrega de nuevos métodos matemáticos, a través de cursos breves.

Existe también en realización un trabajo que aunque figura como programa de extensión es una Investigación sobre los Requisitos de Ingreso a la Universidad, que una vez realizado se pondrá en conocimiento de las autoridades pertinentes para servir de antecedente frente a decisiones que deban tomarse al respecto.

En este trabajo se tratará de probar la hipótesis que "las ponderaciones de los factores no afectan, la selección de los estudiantes que ingresan a la Universidad".

E1 plazo de trabajo se encuentra previsto entre el $1^{\circ}$ de Junio y el 13 de Julio del presente año. 


\section{NOMBRAMIENTO DE NUEVO ACADEMICO}

Con fecha $1^{\circ}$ de Abril de 1982 , se nombró como nuevo académico, de jornada completa de Departamento de Matemática al Sr. Fernando Jorquera Molina, profesor de Matemática (Universidad de Chile, 1967) y Magister en Matemática (Universidad Técnica del Estado, 1973). Antes de ingresar a nuestra Universidad se desempeñó como profesor jornada completa en el Departamento de Matemática de la Universidad de Santiago. E1 Sr. Jorquera tiene 15 años de docencia universitaria $y$ ha aprobado todos los cursos del Programa e Magister en Educación Matemática de la Universidad de Santiago.

Con este nombramiento el Departamento de Matemática de la Universidad del Norte, cuenta entre su personal docente de jornada completa con dos doctores (uno en Análisis Numérico y otro en Estadística), nueve Masters, de los cuales tres lo son en Estadística (CIENES), dos tienen cursos completos de doctorado (Francia y Brasil) y uno se encuentra obteniendo su doctorado en la Universidad de Carolina del Norte (E.E.U.U.). También hay dos licenciados y cinco profesores de Matemática y Física, todos ellos con numerosos cursos a nivel de masters.

\section{REGRESO DEL DR. ARNOLDO PRADO}

En Marzo de este año, el profesor Luis Arnoldo Prado C., finalizó con total éxito sus estudios doctorales en la Universidad Complutense de Madrid. E1 Dr. Prado ha sido recibido con gran satisfacción y alegría, pues estamos conscientes que su participación en el trabajo académico 
significa un valioso, equilibrado y oportuno aporte a la comunidad universitaria.

Ante el interés del Departamento por conocer algunos detalles del perfeccionamiento en España, el Doctor Prado nos ha informado lo siguiente:

1. ¿Cuál es el plan de estudios que culmina con la obtención del Doctorado y cuál fue su modo de enfrentar10 ?

Las normas vigentes en la Facultad contemplan dos tipos de estudios, uno conducente al título de Licenciado en Ciencias Matemáticas y otro conducente al título de Doctor. El primero comprende un plan de 5 años, diferenciado a partir del $7^{\circ}$ semestre para las tres especializaciones que se imparten actualmente: Estadística Matemática, Ecuaciones Funcionales, Educación Matemática. Se accede a este tipo de estudios desde la enseñanza media, previa etapa de selección. El segundo tipo de estudios es el doctorado, con un plan de cuatro asignaturas, pudiendo realizarse un máximo de tres por año lectivo, y la presentación de un trabajo de investigación inédito llamado tésis doctoral. Se accede a este plan desde la Licenciatura y previa obtención, mediante exámen (=reválida) o presentación de un trabajo de investigación (=tesina), del grado de Licenciado, el cual a su vez supone la obtención previa del título de Licenciado.

Se estima que el tiempo medio para la preparación y presentación de una tésis doctoral, incluyendo el período de asignatura, en más o menos 4 años. 
De acuerdo a las disposiciones vigentes durante el año lectivo 1978-1979, debí asimilar los estudios realizados en Chile a los equivalentes de la Licenciatura en Ciencias Matemáticas española. El trámite implica una etapa técnico-administrativa en organismos del gobierno español y una etapa académica; en mi caso debí rendir una exámen, denominado "exámen de conjunto", sobre materias de los planes de la Licenciatura, cuyo resultado positivo me declaró apto para convalidar mis estudios por los de la Licenciatura. Posteriormente, previa aceptación de la autoridad competente y el auspicio de un profesor del Departamento, presenté tesina para optar al grado de Licenciado. El resultado positivo de esta presentación me habilitó para inscribirme en el plan conducente al título de doctor.

A partir de ese momento realicé las obligaciones que me condujeron finalmente a la presentación de la tésis doctoral denominada "Enfoque bayesiano para el "valor de información" en juegos".

2. ¿Cuál es la infraestructura que sustenta el post-grado en España?

La Facultad se organiza en Departamentos, entre los cuales figura el de Estadística Matemática, Ecuaciones funcionales y otros cuyo nombre no retengo en estos momentos. Se cuenta con una biblioteca general para la $\mathrm{Fa}-$ cultad con abundante material bibliográfico actualizado y un número alto de revistas especializadas editadas en los principales centros científicos del mundo. Además, cada Departamento cuenta con recursos bibliográficos de alta especialización. 
Además existe el "Consejo de Investigaciones científicas", organismo estatal que centraliza la actividad científica en general y bajo el cual se cobijan los distintos Institutos, los cuales a su vez cobijan las reuniones especializadas. En cada Instituto existe una biblioteca general y una hemeroteca para cada sección.

Se puede añadir a la infraestructura de apoyo a la investigación el "Centro de Documentación" organismo fiscal que organiza la información científica. Mediante los servicios de este organismo se puede solicitar, vía mecanismos autorizados, información a centros afines de otro lugares del mundo.

Es corriente en España la realización de conferencias y encuentros de especialistas de prestigio internacional. E1 personal de la Facultad participa activamente en estos encuentros ya sea como conferencistas o mediante la presentación de trabajos. Es importante observar que la mayoría de los miembros del Departamento de Estadística Matemática es gente joven, yo diría no superior a los 35 años. En estos momentos se cuenta entre el personal de la Facultad, personalidades de relevancia internacional como Don Sixto Ríos, Don Francisco Azorín Poch y otros, así también existe un número creciente de profesores jóvenes que publican en las principales revistas de la especialidad en el ámbito europeo. 